\title{
Intracameral Injection of Bevacizumab for the Treatment of Neovascular Glaucoma
}

\author{
Armin Wolf $^{a}$ Burkhard von Jagow ${ }^{a, b}$ Michael Ulbig ${ }^{a}$ Christos Haritoglou ${ }^{a}$

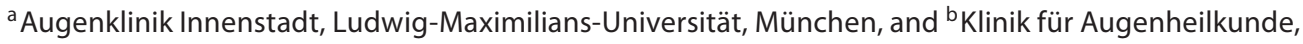 \\ Johann Wolfgang Goethe-Universität, Frankfurt, Germany
}

\section{Key Words}

Anterior chamber $\cdot$ Avastin $^{\circledR} \cdot$ Bevacizumab - Eye .

Neovascularization $\cdot$ Secondary glaucoma $\cdot$ VEGF

\begin{abstract}
Purpose: To assess the duration of the effect of intracameral bevacizumab in patients presenting with rubeosis iridis and neovascular glaucoma (NVG). Methods: Retrospective analysis of 24 consecutive eyes of 24 patients with decompensated NVG $(>21 \mathrm{~mm} \mathrm{Hg})$ treated with a single intracameral injection of bevacizumab over a minimum follow-up of 6 months. The endpoint of the study was the need for retreatment due to recurrence of raised intraocular pressure (IOP). Secondary outcome was the course of visual acuity (VA) and IOP over 6 months. Results: A Kaplan-Meier calculation revealed a mean duration of the treatment effect of $23 \pm 4.4$ days. Compared to mean IOP before treatment $(26.3 \mathrm{~mm}$ $\mathrm{Hg})$, decreases to $17.5 \mathrm{~mm} \mathrm{Hg}$ at 1 week after treatment $(\mathrm{p}<$ $0.002)$ and to $17.1 \mathrm{~mm} \mathrm{Hg}(\mathrm{p}<0.005)$ at 6 months following a single injection were seen. At 6 months, additional treatment was performed in $87.5 \%(n=21)$ of eyes. VA remained stable or improved in $75 \%(n=18)$ of all cases. Conclusion: The IOP-lowering effect of intracameral bevacizumab can be seen 1 week after the injection, but is limited to a period of approximately 3 weeks. However, the fast and effective response to intracameral bevacizumab injection opens a time window for additional treatments, which are often necessary.

Copyright $\odot 2011$ S. Karger AG, Basel
\end{abstract}

\section{KARGER}

Fax +41613061234 E-Mail karger@karger.ch www.karger.com
(C) 2011 S. Karger AG, Basel 0030-3755/11/2262-0051\$38.00/0

Accessible online at:

www.karger.com/oph

\section{Introduction}

Neovascular glaucoma (NVG) is a severe condition resulting from ischemia induced by various ocular diseases, such as proliferative diabetic retinopathy, retinal vein occlusion, radiation retinopathy, and chronic uveitis $[1$, 2]. In NVG, neovascularization of the iris and anterior chamber angle - mediated by vascular endothelial growth factor (VEGF) - leads to elevated intraocular pressure (IOP) [3-6]. Production of VEGF in NVG eyes is triggered by hypoxia of the retina $[2,5]$. In order to lower the raised IOP in patients with NVG, both surgical and medical treatment strategies have been established, such as cyclophotocoagulation or the application of IOP-lowering drops [1]. Retinal ischemia and the resulting increase in intravitreal VEGF levels, which represent the leading pathomechanism in the development of NVG, are reduced by performing panretinal laser photocoagulation (PRP) or cryocoagulation.

However, at least in the short term, photocoagulation alone may not be enough to prevent the progression of NVG - especially in cases with severe and rapid progression of NVG [1]. Despite several treatment modalities being available, visual prognosis is poor and the risk of requiring enucleation in cases of painful amaurosis is high.

\section{A.W. and B.v.J. contributed equally.}

Armin Wolf, MD

Ophthalmology Department, Ludwig Maximilians University

Mathildenstrasse 8

DE-80336 Munich (Germany)

Tel. +49 895160 3811, E-Mail armin.wolf@med.uni-muenchen.de 
Several studies have shown that the monoclonal antibody bevacizumab can be applied intravitreally as well as intracamerally to reduce neovascularization of the iris and thus lower IOP [7-11], but recurrences of elevated IOP are frequent [11]. Therefore, intraocular bevacizumab may be considered as a temporary treatment that reduces IOP and thereby opens the window for additional treatments which could not be performed initially [12].

Although treatment effects have been described by several authors, little is known about the duration of the effect following a single injection of bevacizumab and whether additional treatments should be applied. While such data has been recently published for the intravitreal application [11, 13], no data is available for a single intracameral application of bevacizumab. The aim of this retrospective study was to observe the sustainability of the effect of a single intracameral injection of bevacizumab over time in patients presenting with decompensated NVG.

\section{Patients and Methods}

The authors retrospectively reviewed 32 patient files and included 24 consecutive eyes of 24 patients who received intracameral bevacizumab (Avastin ${ }^{\circledR}$; Gentech, Inc. South San Francisco, Calif., USA) between September 2006 and December 2007 for the treatment of decompensated NVG over a minimum follow-up of 6 months. NVG was defined as IOP $>21 \mathrm{~mm} \mathrm{Hg}$, with the presence of rubeotic vessels in the anterior chamber angle or corneal edema and obvious rubeosis iridis in cases where the anterior chamber angle was not accessible. The primary ocular diseases that led to the development of NVG are listed in table 1. An additional treatment at any time point was defined as the endpoint of this retrospective analysis.

All eyes had follow-up examinations at 1 week, 1 month, and 6 months after intracameral bevacizumab application. The records were reviewed for best corrected visual acuity (BCVA; measured by ETDRS charts if available), IOP (measured by Goldmann applanation tonometry), and the number of glaucoma medications. All patients included had previously been treated by PRP. In some cases, this treatment could not be continued due to corneal edema. Intracameral as opposed to intravitreal injection was preferred, as we assumed that higher drug concentrations would be achieved in the anterior segment.

Informed consent was obtained from each patient prior to the operation. All patients were informed about the 'off-label' use of bevacizumab and the experimental approach. This study was approved by the local institutional review board.

A $50-\mu \mathrm{l}$ aliquot of bevacizumab $(25 \mathrm{mg} / \mathrm{ml})$ was prepared for each patient. After aseptic preparation (10\% povidone-iodine solution) and topical anesthetic eye drops (propacain 2\%), $0.05 \mathrm{ml}$ bevacizumab solution was injected using a 27 -gauge needle at the limbus in the temporal quadrant. Standard postoperative treatment included topical antibiotic eye drops (Ofloxacin, Floxal ${ }^{\circledR}$, Alcon, Hünenburg, Switzerland) for 4 days. IOP and VA were
Table 1. Primary ocular diseases that led to NVG in our sample

\begin{tabular}{lcl}
\hline & Eyes $(\mathrm{n}=24)$ & $\%$ \\
\hline Venous occlusion & 14 & 58 \\
Diabetic retinopathy & 4 & 17 \\
Radiation retinopathy & 3 & 12.5 \\
Severe bulb trauma & 3 & 12.5 \\
\hline
\end{tabular}

monitored in all cases at 1 week and 1 month postoperatively, and at the clinical endpoint. Thereafter, the patients were reviewed at differing time spans. A rise in IOP was interpreted as a recurrence of NVG requiring further treatment.

We defined the need for additional treatment, such as cryocoagulation or completion of PRP as well as glaucoma procedures (e.g. cyclophotocoagulation), as the clinical endpoint of the analysis. The time interval since the intracameral drug application and the nature of the surgical intervention were documented.

Data were collected using a Microsoft Excel spreadsheet. The statistical software BIAS (Ackermann, Frankfurt, Germany) was used to compare means of continuous variables using Student's $t$ test. Non-parametric values were compared with the paired Mann-Whitney-White test. Values of $\mathrm{p} \leq 0.05$ were considered statistically significant. For statistical analysis, VA was transformed to LogMAR VA according to Colenbrander, as described by Duane [14]. For graphical analysis, SPSS was used (SPSS Inc. Chicago, Ill., USA).

\section{Results}

Of the 24 patients, 5 were female and 19 were male. The median age was 74 years (range $31-88$ years).

All eyes had been treated previously with PRP, and 12 eyes had undergone photocoagulation $(\mathrm{n}=8)$ or cryocoagulation $(\mathrm{n}=4)$ of the ciliary body. In 16 eyes, completion of PRP was prevented by corneal edema.

Anterior chamber injection was performed without complication in all cases.

\section{Development of Rubeotic Vessels}

Before intracameral drug application, the anterior chamber could be evaluated in 8 patients. All of these patients showed evident rubeotic vessels in the anterior chamber angle of more than $270^{\circ}$ ( 6 patients with evident 360-degree angle involvement).

Iris neovascularizations completely resolved clinically in 22 eyes (92\%) 1 week postoperatively; in 2 eyes ( $8 \%)$ marked neovascularization in the anterior chamber angle was still present. 


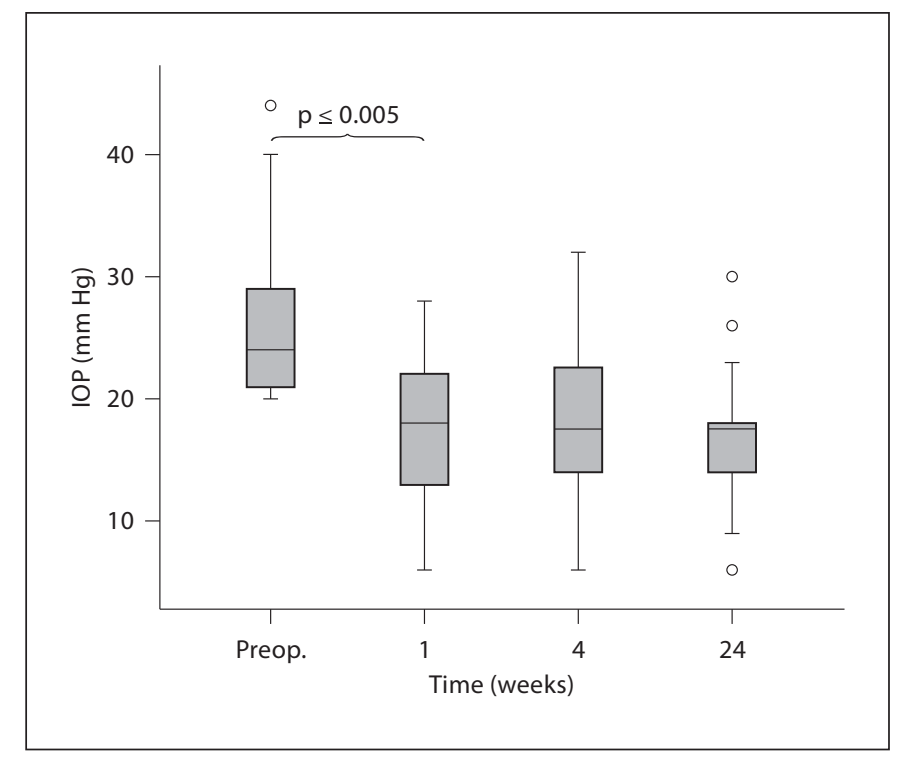

Fig. 1. Median IOPs showing a significant reduction over time $(\mathrm{p}<0.005$, Student's $\mathrm{t}$ test). Kaplan-Meier calculation of the survival time revealed an estimated median of $30 \pm 6.4$ days.

During follow-up, a recurrence of rubeotic vessels of the iris occurred in all eyes (100\%) at different intervals. The median interval to recurrence of iris neovascularization was $18 \pm 57$ days (range 8-160 days).

\section{Development of IOP}

The mean IOP before intracameral injection of bevacizumab was $26.3 \pm 6.6 \mathrm{~mm} \mathrm{Hg}$ (range $21-44 \mathrm{~mm} \mathrm{Hg}$ ), despite topical and systemic IOP-lowering medication. Over all patients, a decrease in IOP to $17.5 \pm 5.3 \mathrm{~mm} \mathrm{Hg}$ $(\mathrm{p}<0.002)$ was noted at 1 week after treatment, and to $17.1 \pm 5.3 \mathrm{~mm} \mathrm{Hg}(\mathrm{p}<0.005)$ after 6 months (fig. 1). A lowered IOP was seen in $70 \%$ of the treated eyes and allowed for the reduction/stoppage of oral glaucoma treatment. In these eyes, an average baseline IOP of $31.13 \pm$ $8.3 \mathrm{~mm} \mathrm{Hg}$ and a decrease to $16.0 \pm 5.2 \mathrm{~mm} \mathrm{Hg}$ after 1 week were noted. In $30 \%$ of treated eyes, IOP remained unchanged.

Along with the decrease in IOP after treatment, the percentage of patients requiring oral glaucoma medication decreased from $33 \%(\mathrm{n}=8)$ before treatment to $21 \%$ $(\mathrm{n}=5)$ at 1 week, and further to $13 \%(\mathrm{n}=3)$ at time of retreatment ( $\mathrm{p} \leq$ 0.001, exact Fisher test, crosstab).

However, the number of topical medications was not affected during follow-up (preoperatively: median $=3$, mean $=2.8$; at last follow-up: median $=3$, mean $=2.8$ ) .

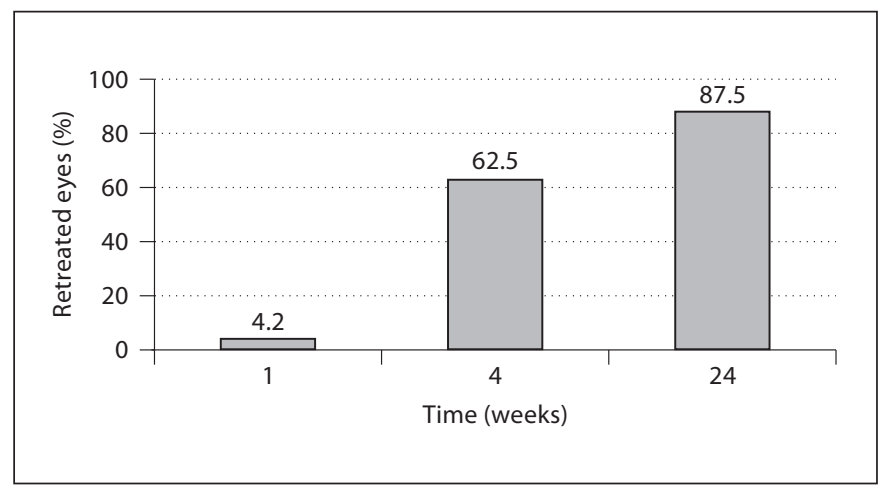

Fig. 2. Proportion of eyes undergoing additional treatment after intracameral bevacizumab. By 6 months, almost all eyes had undergone additional treatment such as completion of PRP or photo- or cryocoagulation of the ciliary body.

None of the eyes required enucleation due to uncontrollably painful glaucoma.

\section{Need for Retreatment after Intracameral Bevacizumab}

At 1 week after treatment with intracameral bevacizumab, further treatment due to persistently increased IOP was performed in 1 eye (4.2\%). At 1 month, additional treatment was performed in $62.5 \%(\mathrm{n}=15)$ of all cases. This number increased to $87.5 \%(\mathrm{n}=21)$ at 6 months (fig. 2). In the remaining cases $(n=3)$, IOP could be controlled by topical treatment and did not show a recurrence of NVG (fig. 2).

The median time interval to additional treatment due to increased IOP was $26 \pm 31$ days (range 2-150 days,). A Kaplan-Meier calculation of the survival time revealed an estimated median of $30 \pm 6.4$ days (estimated mean: $49.2 \pm 11.3$ days).

The most frequent additional treatment consisted of PRP, which was carried out in 12 eyes (50\%). Of note, PRP could not be performed in any of these eyes prior to the intracameral bevacizumab application due to marked IOP-induced corneal edema, which significantly regressed following the IOP-lowering effect of intracameral bevacizumab. Cryocoagulation of the ciliary body and the retina was performed in 3 eyes, and 2 eyes under- 
went pars plana vitrectomy (8\%). Reasons for the pars plana vitrectomies were a tractive retinal detachment in a patient with proliferative diabetic retinopathy 137 days after intracameral bevacizumab and a persistent vitreous hemorrhage in a patient with central retinal vein occlusion 21 days after intracameral bevacizumab (both cases combined with endolaser photocoagulation). A retreatment with intracameral bevacizumab was applied in 4 eyes (16\%). In 3 of the 4 eyes, the intracameral reinjection of bevacizumab was combined with cyclophotocoagulation.

\section{Development of VA}

Preoperatively, BCVA (logMAR) was +0.2 in 1 eye, between +1.3 and +0.7 in 3 eyes, and counting fingers in the remaining 20 eyes. Overall, 73\% (18 eyes) revealed no change in VA during follow-up. In 5 eyes, a decreased VA at the end of follow-up was noted. Three eyes showed an improved VA compared to baseline at 1 week after treatment, with 1 eye maintaining an improved BCVA until the end of follow-up at 6 months.

Mean BCVA remained unchanged during the course of the study and was $1.9 \pm 1.1 \log$ MAR before intracameral bevacizumab, 1 week later $(1.9 \pm 1.0 \log \mathrm{MAR})$, and at 6 months $(1.9 \pm 1.0 \log$ MAR $)$.

\section{Discussion}

NVG is not only a sight-threatening but also a painful condition that may warrant enucleation of the eye. NVG may be the result of different retinal diseases, such as proliferative diabetic retinopathy or retinal vein occlusion. As shown by the Diabetic Retinopathy Study [15] and others [16-18], panretinal photocoagulation represents an effective treatment option in NVG by reducing retinal ischemia and consequently lowering intravitreal VEGF levels. However, according to clinical experience, it may take weeks until the IOP-lowering effect of such laser treatments can be measured. Additionally, retinal photocoagulation is sometimes rendered impossible due to corneal edema resulting from elevated IOP.

We hypothesized that potential benefits from intracameral injection of anti-VEGF drugs (such as bevacizumab) may be derived from the far earlier treatment $[7$, $8,19]$ and the fact that it can be performed irrespective of the presence of media opacities. In addition, as advanced stages of NVG are often refractory to PRP alone, we assumed that the application of an additional treatment modality may positively influence these severe cases, as it has been demonstrated by Ehlers et al. [20] that a combination of PRP with intravitreal bevacizumab increased the speed of regression of neovascularization when compared to PRP alone.

Bevacizumab is a full-length humanized antibody that binds all isoforms of VEGF. Recent case reports have demonstrated the use of off-label intravitreal bevacizumab injections to treat NVG [7-11]. These reports included relatively large numbers of cases, and showed shortand mid-term efficacy and safety to reduce rubeosis iridis and NVG [7-11]. However, as seen in clinical practice, there is a recurrence of increased IOP in many bevacizumab-treated eyes and additional treatments may become necessary. In contrast, the results of Wakabayashi et al. [11] and Jonas et al. [13] imply that additional treatment may not be necessary in all eyes treated with intravitreal bevacizumab. A treatment effect of an intravitreal injection lasting up to an average of 58 days in NVG with open anterior chamber angle has been reported [11]. In contrast to these reports on intravitreal bevacizumab injections, few data are available on the onset and efficacy of a single intracameral bevacizumab injection and on the rate of recurrences in terms of IOP increase following this treatment.

Our data show that intracameral bevacizumab is followed by a rapid decrease in IOP in the majority of cases. In addition, we were able to demonstrate that there is a recurrence of NVG in the majority of eyes over time, and that the time point of recurrence is variable as indicated by the large standard deviation. The earliest recurrence of increased IOP was noted as early as 8 days after intracameral bevacizumab application. The earliest additional treatment was performed 2 days after intracameral bevacizumab application. As shown by the KaplanMeier analysis, a recurrence is found after a period of 4 weeks.

However, this obviously shorter effect (as compared to intravitreal injection) may be derived from a shorter halflife: little is known about the half-life duration of intracameral bevacizumab. In general, one may assume that intracamerally applied drugs have a shorter half-life than intravitreally applied drugs, as described for other drugs such as voriconazole [21,22]. The half-life of intravitreal bevacizumab was calculated to be 4.32 days in an animal model and 9.8 days in humans [23, 24].

Interestingly, at the 6-month follow-up, retreatment was necessary in the majority of eyes. This is in accordance with recent data published by Jonas et al. [13]. Our results indicate that retreatment - if it becomes necessary - is performed within 3 weeks. However the wide 
range in time until retreatment (of up to 150 days) indicates that all eyes previously treated with intracameral bevacizumab should be followed closely in order to identify those patients where a retreatment is not necessary.

As described before [7], the efficacy of a single intracameral injection was impressive: a single application of the drug caused dramatic reduction in the biomicroscopically visible rubeotic vessels in $92 \%$ of the cases. As in previous studies on intravitreal bevacizumab, no adverse events related to intracameral injection were noted.

We failed to identify prognostic factors concerning the recurrence of NVG. Wakabayashi et al. [11] reported that an IOP-lowering effect of intravitreal bevacizumab injections depends on the status of the anterior chamber angle, with a decrease in IOP seen only in cases with open anterior chamber angle and no effect seen in eyes with closed angle. In our analysis, the angle could only be assessed in a limited number of cases due to corneal edema. Therefore, we cannot comment on this prognostic factor in our study. However, the fact that at 6 months the majority of patients had undergone additional surgeries (including completion of PRP) may allow us to conclude that the eyes included in our study represented end-stage NVG with - at least to some extent - closed angles.

In our study, we were able to stabilize VA in the majority of patients and none of the patients underwent enucleation during follow-up. Nevertheless, in 20\% (5 eyes) visual function further deteriorated, which may be related to glaucoma damage or progression of the concomitant retinopathy.
We are aware of the limitations of our study, including its retrospective design and limited follow-up, as well as a heterogenous group of patients with respect to the underlying diseases. Therefore, it seems difficult to compare the efficacy of an intracameral bevacizumab injection as performed in our patients to studies in which the drug was applied intravitreally [11]. A prospective comparative study addressing this point may be useful to further elucidate this issue.

The cases presented herein demonstrate the potential of an intracameral bevacizumab injection to lower IOP in NVG patients, reduce the need for IOP-lowering drugs, and achieve better conditions for additional treatments such as PRP (as all patients who underwent completion of PRP could not have done this before the intracameral bevacizumab injection due to corneal edema). We have learned from our investigation that such treatments mostly become necessary at approximately 20 days after intracameral bevacizumab injection. Regular measurements of IOP following the intracameral injection appear mandatory in order to identify those few patients in whom IOP could be stabilized by a single intracameral injection and those who require earlier additional treatments than indicated above.

\section{Disclosure Statement}

No author has any proprietary interest or conflict of interest related to any product mentioned in the article.

\section{References}

1 Sivak-Callcott JA, O’Day DM, Gass JD, Tsai JC: Evidence-based recommendations for the diagnosis and treatment of neovascular glaucoma. Ophthalmology 2001;108:17671776, quiz 1777, 1800.

2 Shazly TA, Latina MA: Neovascular glaucoma: etiology, diagnosis and prognosis. Semin Ophthalmol 2009;24:113-121.

3 Aiello LP: Vascular endothelial growth factor and the eye: biochemical mechanisms of action and implications for novel therapies. Ophthal Res 1997;29:354-362.

4 Aiello LP, Avery RL, Arrigg PG, et al: Vascular endothelial growth factor in ocular fluid of patients with diabetic retinopathy and other retinal disorders. New Engl J Med 1994;331:1480-1487.
5 Aiello LP, Wong JS: Role of vascular endothelial growth factor in diabetic vascular complications. Kidney Int 2000;77:S113S119.

6 Tripathi RC, Li J, Tripathi BJ, Chalam KV, Adamis AP: Increased level of vascular endothelial growth factor in aqueous humor of patients with neovascular glaucoma. Ophthalmology 1998;105:232-237.

7 Batioglu F, Astam N, Ozmert E: Rapid improvement of retinal and iris neovascularization after a single intravitreal bevacizumab injection in a patient with central retinal vein occlusion and neovascular glaucoma. Int Ophthalmol 2008;28:59-61.

8 Grisanti S, Biester S, Peters S, Tatar O, Ziemssen F, Bartz-Schmidt KU: Intracameral bevacizumab for iris rubeosis. Am J Ophthalmol 2006;142:158-160.
9 Davidorf FH, Mouser JG, Derick RJ: Rapid improvement of rubeosis iridis from a single bevacizumab (Avastin) injection. Retina 2006;26:354-356.

10 Kitnarong N, Chindasub P, Metheetrairut A: Surgical outcome of intravitreal bevacizumab and filtration surgery in neovascular glaucoma. Adv Ther 2008;25:438-443.

11 Wakabayashi T, Oshima Y, Sakaguchi H, et al: Intravitreal bevacizumab to treat iris neovascularization and neovascular glaucoma secondary to ischemic retinal diseases in 41 consecutive cases. Ophthalmology 2008; 115 : 1571-1580, 1580 e1571-e1573.

12 Luke J, Luke M, Grisanti S: Antiangiogenic treatment for neovascular glaucoma and after filtering surgery (in German). Ophthalmologe 2009;106:407-412. 
13 Jonas JB, Golubkina L, Libondi T, Rensch F: Intravitreal bevacizumab for neovascular glaucoma. Acta Ophthalmologica 2009; 88:e22-e23.

14 Duane T: Clinical Ophthalmology. New York, Lippincott, Williams \& Wilkins, 2001.

15 Photocoagulation treatment of proliferative diabetic retinopathy: the second report of diabetic retinopathy study findings. Ophthalmology 1978;85:82-106.

16 Augsburger JJ, Roth SE, Magargal LE, Shields JA: Panretinal photocoagulation for radiation-induced ocular ischemia. Ophthal Surg 1987; 18:589-593.
17 Magargal LE, Brown GC, Augsburger JJ, Donoso LA: Efficacy of panretinal photocoagulation in preventing neovascular glaucoma following ischemic central retinal vein obstruction. Ophthalmology 1982;89:780784 .

18 Tasman W, Magargal LE, Augsburger JJ: Effects of argon laser photocoagulation on rubeosis iridis and angle neovascularization. Ophthalmology 1980;87:400-402.

19 Chalam KV, Gupta SK, Grover S, Brar VS Agarwal S: Intracameral Avastin dramatically resolves iris neovascularization and reverses neovascular glaucoma. Eur J Ophthalmol 2008;18:255-262.

20 Ehlers JP, Spirn MJ, Lam A, et al: Combination intravitreal bevacizumab/panretinal photocoagulation versus panretinal photocoagulation alone in the treatment of neovascular glaucoma. Retina 2008;28:696-702.
21 Shen YC, Wang MY, Wang CY, et al: Pharmacokinetics of intracameral voriconazole injection. Antimicrob Agents Chemother 2009;53:2156-2157.

22 Shen YC, Wang MY, Wang CY, et al: Clearance of intravitreal voriconazole. Invest Ophthalmol Vis Sci 2007;48:2238-2241.

23 Bakri SJ, Snyder MR, Reid JM, Pulido JS, Singh RJ: Pharmacokinetics of intravitreal bevacizumab (Avastin). Ophthalmology 2007; 114:855-859.

24 Krohne TU, Eter N, Holz FG, Meyer CH: Intraocular pharmacokinetics of bevacizumab after a single intravitreal injection in humans. Am J Ophthalmol 2008;146:508-512. 